\title{
Effects of healthy pseudopregnant milieu on development of two-cell subdiabetic mouse embryos
}

\author{
J. Veselá, P. Rehák, V. Baran and J. Koppel \\ Institute of Animal Physiology, Slovak Academy of Sciences, Košice, Slovak Republic
}

\begin{abstract}
Female mice were injected with a single dose of streptozotocin ( $65 \mathrm{mg} \mathrm{kg}^{-1}$ ) 14-17 days before fertilization to investigate the significance of impaired insulin secretion induced by subdiabetic streptozotocin treatment on preimplantation embryo development. Subdiabetic mice (streptozotocin-treated) had significantly different glucose tolerance from that of control animals, despite similar basal glycaemia. Morphological analysis of preimplantation embryos collected on day 2 of pregnancy revealed no significant changes in the number of two-cell embryos recovered from streptozotocin-treated females compared with controls. Two-cell embryos were transferred into the oviducts of healthy, synchronous pseudopregnant females and recovered 24-28 h later. Morphological evaluation revealed a significantly greater percentage of degenerated embryos from streptozotocin-treated females than from control females. Morphological analysis of preimplantation embryos collected on day 2.5 of pregnancy revealed no significant changes in the number of two- to four-cell embryos recovered from streptozotocin-treated females compared with controls, but there was a significant increase in the number of degenerated embryos in streptozotocin-treated females that did not receive insulin therapy. Insulin (1-1.5 iu per $100 \mathrm{~g}$ ) administered twice a day to streptozotocin-treated mice significantly improved the altered development of embryos in both experiments. It is possible that the impaired insulin secretion in female mice adversely affected the growth of preimplantation embryos. Almost half of the morphologically normal two-cell embryos isolated from subdiabetic females were incapable of development to the eight-cell stage even in a non-diabetic maternal environment. The morphologically distinct degenerative changes were first detected at the time of the second mitotic cleavage.
\end{abstract}

\section{Introduction}

The development of preimplantation mammalian embryos is controlled by specific maternal and embryonic genes, as well as by hormones and a continuing supply of energy from the mother. It is well established that insulin plays the role of a growth factor for many rapidly proliferating cells and its effects on preimplantation embryo development are of interest.

There is increasing evidence to support the involvement of insulin in the control of early embryo development. The stimulation of protein synthesis by insulin in vitro at the time of embryo compaction coincides with the appearance of insulin receptors on compacting eight-cell embryos and a switch in energy dependence from lactate and pyruvate to glucose (Gardner and Leese, 1988; Harvey and Kaye, 1991). Little work has been done on the effects of impaired maternal insulin secretion induced by diabetes on the preimplantation embryo. Experimental studies established that rodents with chemically induced diabetes (treatment with streptozotocin or alloxan) are a suitable model for studying the early deleterious effects of maternal diabetes on preimplantation embryo development (Diamond et al., 1989; Beebe and Kaye, 1991; De Hertogh et al.,
1992). Observations in rats (Vercheval et al., 1990) showed that preimplantation embryos are altered by a single subdiabetogenic dose of streptozotocin. We recently observed that in female mice the subdiabetic state induced by streptozotocin resulted in impaired preimplantation embryo development up to the eight-cell stage that could be partly prevented by insulin treatment (Veselá et al., 1993). These observations indicate a key role for insulin during preimplantation development.

The aim of this study was to identify the exact stage of embryo development at which the deleterious effects of streptozotocin treatment are manifested. We, therefore, investigated the development of preimplantation embryos isolated at the two-cell stage from subdiabetic mice and transferred into the oviducts of healthy synchronous pseudopregnant females and recovered $24-28 \mathrm{~h}$ later. The morphological development of preimplantation embryos isolated from subdiabetic mothers on day 2.5 of pregnancy was also investigated.

\section{Materials and Methods}

\section{Animals}

The study was conducted using donor female mice of the inbred BALB/C strain (Velaz, Prague), 6-8 weeks old and 
18-20 g body weight. Recipient females were of the outbred ICR strain, 8-10 weeks old, and 20-22 g body weight. The mice had free access to food (DOS $2 \mathrm{~b}$ diet, Velaz, Prague) and water. Animals were maintained in a $12 \mathrm{~h}$ light: $12 \mathrm{~h}$ dark cycle (lights on at $06.00 \mathrm{~h}$ ). Only females with confirmed normal 4-5 day oestrous cycles were used.

\section{Induction of subdiabetic state}

Streptozotocin (Serva, Heidelberg) was dissolved in a sterile sodium citrate buffer $\left(0.1 \mathrm{~mol} \mathrm{I}^{-1}, \mathrm{pH} \mathrm{4.5)}\right.$ and injected within 5 min of preparation into donor females. Mice received a single i.p. subdiabetogenic dose of streptozotocin $\left(65 \mathrm{mg} \mathrm{kg}^{-1}\right.$ body weight) 14-17 days before mating.

Ultralente insulin (Pur-Insulin-Superdep: Spofa, Prague) was injected s.c. twice a day at a dose of 1-1.5 iu per $100 \mathrm{~g}$ body weight starting 6 days after streptozotocin treatment. Control animals were injected initially with citrate buffer and then twice a day with $154 \mathrm{mmol} \mathrm{NaCl}^{-1}$. Blood glucose determinations were made using a glucose oxidase method (Oxochromglucose: Lachema, Brno). Eleven days after streptozotocin administration, the animals in each group (fasted overnight) received an i.p. injection of $10 \%$ glucose solution at $1 \mathrm{~g} \mathrm{~kg}^{-1}$ body weight. Blood samples (taken from the tail vein) were obtained immediately before and $30 \mathrm{~min}$ after glucose administration.

\section{Embryo collection}

Spontaneous ovulation was used to rule out possible effects of exogenous hormones used in superovulation. Donor females were kept with males of the same strain overnight. If a vaginal plug was found on the following morning, this was considered as day 1 of pregnancy. Animals were excluded from the experiments after four unsuccessful matings. Animals were killed by cervical dislocation.

In the first experiment, preimplantation embryos were collected from streptozotocin-treated and control donor mice on day 2 of pregnancy. Experimental bias was avoided by performing two independent series and pooling the results. Only healthy two-cell embryos without any signs of degeneration were used for transfer. The interval between flushing and transfer did not exceed $15 \mathrm{~min}$. Ham's F-10 media with Hepes (Sigma, St Louis, MO) containing 10\% (v/v) bovine fetal serum (Veterinary University, Brno), was used for flushing and transfer.

Pseudopregnancy was induced in recipient females by mating them with vasectomized males of the same strain of proven fertility. Recipients were at day 1 of pseudopregnancy at the time of transfer and were anaesthetized by an i.p. injection of sodium pentobarbitone (Pentobarbital: Spofa, Prague, $0.06 \mathrm{mg}$ $\mathrm{g}^{-1}$ body weight). Two-cell embryos $(n=8-10)$ were transferred in 2-5 $\mu \mathrm{l}$ medium via a drawn-out glass capillary pipette into one oviduct of the recipient; the second oviduct acted as a control. Embryos were recovered, using a dissecting microscope (Technival, Warsaw), by flushing with Ham's F-10 media (Sigma, St Louis, MO) containing 10\% (v/v) bovine fetal serum, 24-28 h after transfer. The recovery rates of embryos after transfer were $72 \%$ in controls, $74 \%$ in the streptozotocin- treated, no insulin group and $88 \%$ in the streptozotocin, insulin-treated group. Embryos were observed by a differential interference contrast microscope (Jenamed, Variant, Jena) and assessed morphologically according to the following criteria: (a) degenerated: embryos with signs of degeneration; (b) abnormal: two- and three-cell embryos and, (c) normal: foureight-cell embryos.

In the second experiment, preimplantation embryos were collected from streptozotocin-treated and control mice on day 2.5 of pregnancy. Embryos were observed using a differential interference contrast microscope and were evaluated morphologically according to the following criteria: (a) degenerated: embryos with signs of degeneration, including unfertilized oocytes and zygotes that could not be morphologically differentiated; (b) two-cell embryos and, (c) three- or four-cell embryos.

\section{Statistical analysis}

Results are given as means $\pm \mathrm{SD}$. Statistical comparisons between groups were performed by two different methods. Chi-square test was used to detect differences in the distribution of preimplantation embryos. Statistical analysis of blood glucose concentrations and number of embryos was performed by ANOVA and Duncan's multiple range test.

\section{Results}

\section{Development stage of embryo at recovery}

There was no significant difference $(P>0.05)$ in the number of two-cell embryos between control and streptozotocintreated mice (Table 1). A similar percentage of degenerated embryos were observed in streptozotocin-treated mice receiving insulin $(29.7 \%)$, streptozotocin-treated mice not receiving insulin $(27.8 \%)$ and control mice $(22.8 \%)$. Mean glycaemia (Table 1) was similar in the control group and in both subdiabetic groups (treated with $1-1.5$ iu insulin per $100 \mathrm{~g}$ body weight twice a day or untreated). However, the glucose tolerance test induced a significant increase in glycaemia $30 \mathrm{~min}$ after glucose administration, in streptozotocin-treated mice compared with control mice $(P<0.01)$.

\section{Morphology of embryos collected from normal pseudopregnant females 24-28 $h$ after transfer}

The distribution pattern of the stage of preimplantation embryos isolated from normal pseudopregnant mothers was different between the streptozotocin-treated and control groups (Table 2, Fig. 1). Subdiabetic mice not receiving insulin treatment had a high percentage $(47.6 \%)$ of degenerated embryos $(P<0.001)$ compared with controls; there was no effect of normal pseudopregnant reproductive environment. Insulin administration, twice a day, resulted in a significant $(P<0.001)$ improvement in the development of preimplantation embryos in the streptozotocin-treated groups comparable with the results of the control group $(P>0.05)$. 
Table 1. Numbers of two-cell embryos and glucose tolerance tests in subdiabetic and control mice

\begin{tabular}{|c|c|c|c|}
\hline Parameter & Controls & STZ + insulin & STZ - insulin \\
\hline Number of mice & 14 & 10 & 20 \\
\hline Total number of embryos & 114 & 91 & 187 \\
\hline Embryos per mouse & $8.14 \pm 2.39$ & $9.1 \pm 2.5$ & $9.35 \pm 2.22$ \\
\hline Percentage of two-cell embryos & 77.2 & 70.3 & 72.2 \\
\hline Percentage of embryos degenerated & 22.8 & 29.7 & 27.8 \\
\hline \multicolumn{4}{|l|}{$\chi^{2}$ test } \\
\hline STZ versus control & & $P>0.05$ & $P>0.05$ \\
\hline Insulin-treated versus no insulin treatment & & \multicolumn{2}{|c|}{$P>0.05$} \\
\hline \multicolumn{4}{|l|}{ Glycaemia (i.p. I g glucose $\mathrm{kg}^{-1}$ ) } \\
\hline Time $0^{a}$ & $4.19 \pm 0.33$ & $4.49 \pm 0.97$ & $3.73 \pm 0.97$ \\
\hline After $30 \mathrm{~min}$ & $5.16 \pm 0.63^{b}$ & $6.51 \pm 1.01^{c}$ & $7.10 \pm 0.82^{c}$ \\
\hline
\end{tabular}

Table 2. Results of transfer of two-cell embryos from subdiabetic and control mice to normal pseudopregnant females and morphological stages of embryos recovered from recipients $24-28 \mathrm{~h}$ later

\begin{tabular}{|c|c|c|c|}
\hline Parameter & Controls & STZ + insulin & $\mathrm{STZ}$ - insulin \\
\hline \multicolumn{4}{|l|}{ Transfer of two-cell embryos } \\
\hline Number of recipient mice & 7 & 7 & 9 \\
\hline Number of embryos transferred & 69 & 59 & 85 \\
\hline Number of embryos per mouse & $9.86 \pm 0.64$ & $8.43 \pm 1.54$ & $9.44 \pm 1.34$ \\
\hline \multicolumn{4}{|c|}{ Distribution pattern of embryos collected $24-28 \mathrm{~h}$ after transfer } \\
\hline Number of embryos recovered & 50 & 52 & 63 \\
\hline Percentage at $4-8$-cell stage & 68 & 78.9 & 47.6 \\
\hline Percentage at $2-3$-cell stage & 22 & 7.8 & 4.8 \\
\hline Percentage of embryos degenerated & 10 & 13.5 & 47.6 \\
\hline \multicolumn{4}{|l|}{$\chi^{2}$ test } \\
\hline STZ versus control & & $P>0.05$ & $P<0.001$ \\
\hline Insulin-treated versus no insulin treatment & & \multicolumn{2}{|c|}{$P<0.001$} \\
\hline
\end{tabular}

Values are means \pm SD.

STZ: streptozotocin-treated mice.

Morphology of embryos recovered from pregnant (day 2.5) control and subdiabetic mice

The numbers of embryos recovered from streptozotocintreated and control mice at day 2.5 did not differ significantly (Table 3, $P>0.05$ ). Statistically significant changes were observed in the stage of development of preimplantation embryos after maternal streptozotocin treatment $(P<0.01)$. A high percentage $(53.1 \%)$ of degenerated embryos was found in streptozotocin-treated females on day 2.5 of pregnancy. Insulin treatment (twice a day) of streptozotocin-treated mice was adequate to ensure normal embryo development in comparison with controls $(P>0.05)$, which was highly significantly different from streptozotocin-treated mice not receiving insulin $(P<0.001)$.

\section{Discussion}

Veselá et al. (1993) observed that the stages of preimplantation embryos recovered on day 3 of pregnancy from mice in a subdiabetic state induced by streptozotocin but not showing any changes in basal glycaemia were significantly different from that of controls and that insulin treatment once a day was only partly effective in restoring embryo development to normal. The study reported here extends these observations and clearly shows that significantly more morphologically normal two-cell embryos recovered from subdiabetic females on day 2 of pregnancy and transferred to healthy pseudopregnant females have degenerated at the time of recovery $24-28 \mathrm{~h}$ later, in comparison with those of the control group. More frequent insulin administration (twice a day) prevented the 


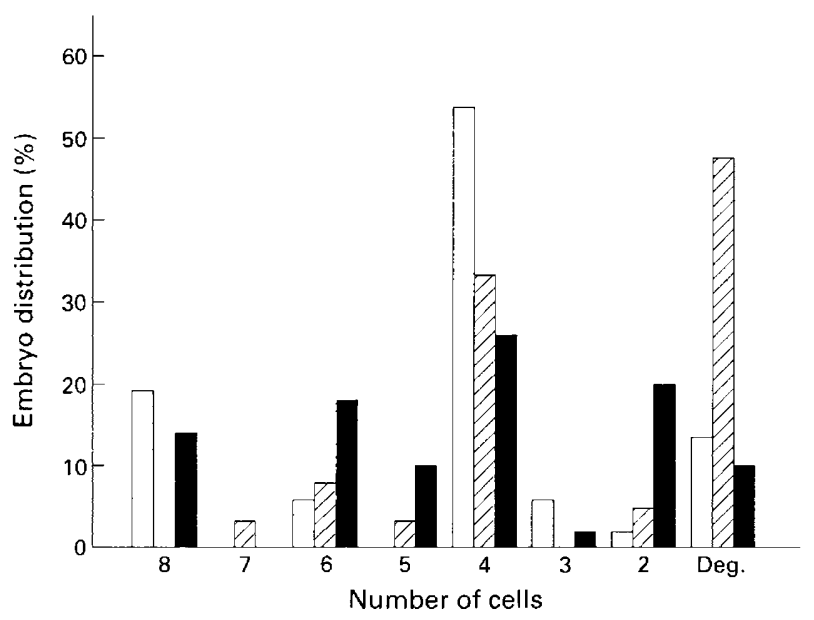

Fig. 1. Percentage of embryos at different stages of development collected from healthy pseudopregnant mice $24-28 \mathrm{~h}$ after transfer from ( $\square$ ) control mice and mice treated with: $(\square)$ streptozotocin and insulin and ( $(\square)$ streptozotocin only. Deg: degenerated embryos.

streptozotocin-induced impairment of preimplantation embryo development. In the second experiment, streptozotocin-treated mice not receiving insulin had a slower embryo development and more embryos had degenerated in comparison with controls. This was apparent by the second mitotic division. It can be assumed that the two-cell embryos were damaged, but this was not apparent under light microscopy.

Spontaneous diabetes in Chinese hamsters did not significantly influence the number of two-cell embryos recovered on day 2.5 of gestation (Funaki and Mikamo, 1983). Beebe and Kaye (1990) reported that streptozotocin-diabetic (190$240 \mathrm{mg} \mathrm{kg}^{-1}$ ) and control mice produced similar numbers of two-cell embryos after superovulation. In the present study, females with spontaneous cycles were used and a comparison of the number of two-cell embryos showed no difference between the subdiabetic and control groups. Similarly in the previous study, we demonstrated that the subdiabetic state in mice which ovulated spontaneously did not result in any significant difference in the number of embryos collected from subdiabetic (streptozotocin-treated) and control mice on day 3 of pregnancy (Veselá et al., 1993). In contrast, Diamond et al. (1989) found fewer two-cell embryos collected from streptozotocin-diabetic mice ( $330 \mathrm{mg} \mathrm{kg}^{-1}$ ) $48 \mathrm{~h}$ after treatment with hCG. These differences may result from the different strains of mice used or the greater dose of streptozotocin.

This evidence suggests that maternal diabetes or the subdiabetic state has no easily detectable effects on ovulation, fertilization and the first embryo cleavage. Nevertheless, almost half of the morphologically normal two-cell embryos isolated from subdiabetic females in the study reported here did not develop up to the eight-cell stage, even in a non-diabetic maternal environment. We demonstrated that a relatively mild degree of impaired insulin secretion alters embryo development up to the eight-cell stage (Veselá et al., 1993). Similarly Vercheval et al. (1990) detected morphological retardation and fewer blastocyst cells on day 5 of pregnancy in subdiabetic rats. Blastocysts collected on day 4 from diabetic mice had fewer cells and a lower rate of protein synthesis than did control embryos (Beebe and Kaye, 1991).

The basis of this altered development is unclear. It was shown, in spontaneously non-obese diabetic (NOD) mice, that uncontrolled diabetes mellitus per se retarded embryo development $72 \mathrm{~h}$ after hCG treatment (Moley et al., 1991), thus excluding a potential toxic effect of streptozotocin on embryo growth. Toxicity caused directly by streptozotocin is unlikely, as maternal insulin treatment improved preimplantation embryo development. Our findings indicate that impaired maternal insulin secretion during the pre- and earliest postconception stage may have a deleterious effect on preimplantation embryo development which persists despite the removal of embryos from the mother and transfer to a non-diabetic milieu. In vivo insulin therapy (twice a day) appeared to reverse this delayed developmental alteration, as there was no significant difference in the embryo stages between control and streptozotocin-treated animals receiving insulin. This observation confirms our previous findings and indicates the importance of normal insulin secretion for preimplantation development.

It appears that changes in maternal glucose may not be directly responsible for the observed impaired embryo

Table 3. Morphological analysis of mouse embryos isolated from subdiabetic mothers and controls on day 2.5 of pregnancy

\begin{tabular}{lccc}
\hline Parameter & Control & STZ + insulin & STZ - insulin \\
\hline Number of mice & 11 & 6 & 18 \\
Total number of embryos & 98 & 58 & 162 \\
Number of embryos per mouse & $8.9 \pm 1.3$ & $9.7 \pm 1.1$ & $9.0 \pm 1.9$ \\
Percentage at 4-cell stage & 4.1 & 6.9 & 5.6 \\
Percentage at 3-cell stage & 11.2 & 5.2 & 3.5 \\
Percentage at 2-cell stage & 52.0 & 70.7 & 53.1 \\
Percentage of embryos degenerated & 32.7 & 17.2 & $P<0.01$ \\
$\chi^{2}$ test & & & $P>0.05$ \\
STZ versus control & & & $P<0.001$ \\
Insulin-treated versus no insulin treatment & & & \\
\hline
\end{tabular}


development. We showed that subdiabetic mice (streptozotocin-treated) have altered glucose tolerance compared with control animals, despite their similar basal glycaemia. It is therefore possible that greater variations in blood glucose could adversely affect development of preimplantation embryos. Blood glucose concentrations sampled every $4 \mathrm{~h}$ for $24 \mathrm{~h}$ did not significantly differ between females treated with streptozotocin but not receiving insulin and control females (data not shown); although a more frequent sampling could be required to reveal subtle and rapid changes in blood glucose. Diamond et al. (1991) showed that a small increase in glucose (12.2 $\mathrm{mmol} \mathrm{^{-1 }}$ ) may retard the development of mouse embryos beginning at the two-cell stage and Chatot et al. (1989) demonstrated that the presence of glucose in the medium could block embryo development (I-4-cell stage) during the first $48 \mathrm{~h}$ of culture. Seshagiri and Bavister (1991) related the toxicity of glucose to hamster 8-cell embryos to the inhibition of oxidative phosphorylation (the Crabtree effect).

The deficiency in insulin secretion could be directly related to altered embryo development. Insulin at physiological concentrations stimulates the metabolism and growth of preimplantation embryos in vitro (Harvey and Kaye, 1988, 1992). However, insulin-receptor mRNA is not expressed until the 8-cell stage in mouse embryos (Schultz et al., 1992) and addition of insulin to the culture medium had no detectable growth-promoting effects on two-cell embryos in vitro (Diamond et al., 1991), excluding a direct influence of insulin on preimplantation embryos. Furthermore, blastocysts from diabetic (streptozotocin-induced) rats were found to utilize glucose at a similar rate to that of normal blastocysts and insulin had no effect on glucose uptake, utilization, incorporation or turnover (Brison and Leese, 1990; Dufrasnes et al., 1993). In addition, Gardner and Leese (1988) concluded that in the mouse preimplantation embryo, insulin does not play a role in glucose transport. Expression of the major insulin-regulated GLUT4 isoform of the glucose transporter has not been demonstrated at any stage of preimplantation development (Aghayan et al., 1992). The role of IGF-I in anomalies of embryo development induced by impaired insulin secretion remains to be clarified. Its importance is emphasized by the observations that diabetes decreases concentrations of circulating IGF-I (reviewed by Herington, 1991) and that IGF-I has growth-stimulating effects on preimplantation embryos, which are mediated up to the 8-cell stage by IGF-2 receptors (Harvey and Kaye, 1992).

In conclusion, impaired insulin secretion in female mice has a negative influence on the growth of preimplantation mouse embryos. Almost half of the morphologically normal two-cell embryos isolated from subdiabetic females are incapable of development up to the 8-cell stage even in a non-diabetic maternal environment. Morphologically distinct degenerative changes are first detected at the time of the second mitotic cleavage. Apparently, marginal changes in the metabolic and hormonal balance in pregnancy can interfere with later embryo development.
This work was supported by grant 61/92 from the Slovak Academy of Sciences.

\section{References}

Aghayan M, Rao LV, Smith RM, Jarett L, Charon MJ, Thorens B and Heyner S (1992) Developmental expression and cellular localization of glucose transporter during mouse preimplantation development Development 115 305-312

Beebe LFS and Kaye PL (1990) Preimplantation development in the streptozotocin-induced diabetic mouse Reproduction, Fertility and Development 4 407-412

Beebe LFS and Kaye PL (1991) Maternal diabetes and retarded preimplantation development of mice Diabetes 40 457-461

Brison DR and Leese HJ (1990) Glucose uptake by embryos from diabetic rats Journal of Reproduction and Fertility Abstract Series 6 Abstract 71

Chatot CL, Ziomek CA, Bavister BD, Lewis JL and Torre I (1989) An improved culture medium supports development of random-bred 1-cell mouse embryos in vitro Journal of Reproduction and Fertility 86 679-688

De Hertogh R, Vanderheyden I, Pampfer S, Robin D and Delcourt J (1992) Maternal insulin treatment improves pre-implantation embryo development in diabetic rats Diabetologia 35 406-408

Diamond MP, Moley KH, Pelicer A, Vaughn WK and DeCherney AH (1989) Effects of streptozotocin- and alloxan-induced diabetes mellitus on mouse follicular and early embryo development Journal of Reproduction and Fertility $861-10$

Diamond MP, Peftway ZY, Logan J, Moley K, Vaughn W and Decherney AH (1991) Dose-response effects of glucose, insulin, and glucagon on mouse preimplantation embryo development Metabolism 40 566-570

Dufrasnes E, Vanderheyden I, Robin D, Delcourt J, Pampfer S and De Hertogh R (1993) Glucose and pyruvate metabolism in preimplantation blastocysts from normal and diabetic rats journal of Reproduction and Fertility 98 169-177

Funaki K and Mikamo K (1983) Developmental-stage-dependent teratogenic effects of maternal sponaneous diabetes in the Chinese hamster Diabetes 32 $637-643$

Gardner DK and Leese HJ (1988) The role of glucose and pyruvate transport in regulating nutrient utilization by preimplantation mouse embryos Development $104423-429$

Harvey MB and Kaye PL (1988) Insulin stimulates protein synthesis in compacted mouse embryos Endocrinology 122 1182-1184

Harvey MB and Kaye PL (1991) Visualization of insulin receptors on mouse pre-embryos Reproduction, Fertility and Development 3 9-15

Harvey MB and Kaye PL (1992) Mediation of the actions of insulin and insulin-like growth factor- 1 on preimplantation mouse embryos in vitro Molecular Reproduction and Development 33 270-275

Herington AC (1991) Insulin-like growth factors: biochemistry and physiology Baillière's Clinical Endocrinology and Metabolism 5 531-551

Moley KH, Vaughn WK, DeCherney AH and Diamond MP (1991) Effect of diabetes mellitus on mouse pre-implantation development journal of Reproduction and Fertility 93 325-332

Schultz GA, Hogan A, Watson AJ, Smith RM and Heyner S (1992) Insulin, insulin-like growth factors and glucose transporters: temporal patterns of gene expression in early murine and bovine embryos Reproduction, Fertility and Development 4 361-371

Seshagiri PB and Bavister BD (1991) Glucose and phosphate inhibit respiration and oxidative metabolism in cultured hamster eight-cell embryos: evidence for the "crabtree effect" Molecular Reproduction and Development 30 105111

Vercheval M, De Hertogh R, Pampfer S, Vanderheyden I, Michiels B, De Bernardi $P$ and De Meyer R (1990) Experimental diabetes impairs rat embryo development during the preimplantation period Diabetologia 33 187-191

Veselá J, Rehák P, Baran V and Koppel J (1993) Subdiabetogenic streptozocin treatment impairs preimplantation development of mouse embryos Physiological Research 42 23-27 